\title{
Evaluación del cumplimiento por parte del personal médico de las indicaciones de transfusión de glóbulos rojos en la población adulta en el Hospital Universitario del Caribe entre 2011 y 2012
}

- Kelman Hanael Ojeda, Álvaro Moreno, Deidy Johana Bustos, María Rosario Fernández, Jaime José Díaz, Mauro Alonso Moreno, Diego Moreno

Universidad de Cartagena, Cartagena (Bolívar).

Contacto: namlek74@hotmail.com

Introducción y objetivos. Las principales indicaciones de transfusiones de glóbulos rojos son síndrome anémico, pacientes críticos hemoglobina $<7 \mathrm{~g} / \mathrm{dl}$, y enfermedad cardiovascular descompensada hemoglobina $<8 \mathrm{~g} / \mathrm{dl}$. El objetivo principal del estudio es evaluar el cumplimiento de las indicaciones de transfusión de glóbulos rojos por parte del personal médico en la población adulta en el Hospital Universitario del Caribe (Cartagena).

Materiales y métodos. Estudio observacional retrospectivo. Realizado en el Hospital Universitario del Caribe entre septiembre de 2011 y agosto de 2012. Se estimaron 361 historias clínicas con margen de error del $5 \%$, un nivel de confianza del $95 \%$, una prevalencia estimada del $62,4 \%$. El análisis estadístico se efectuó con Epi Info versión 7.0. Se evaluaron las principales indicaciones de transfusiones de glóbulos rojos y su cumplimiento en diferentes áreas hospitalarias, basados en la guía de la American Association of Blood Banks. El análisis estadístico incluyó cálculo de proporciones en variables cualitativas, medidas de centralización y dispersión para las cuantitativas.

Resultados. En total 5.706 transfusiones, el 49,2\% de glóbulos rojos, y se incluyeron 420 historias clínicas. Los criterios de transfusión de glóbulos rojos se cumplieron en el $86,43 \%$, distribuidos por servicio así: urgencias, $\mathrm{UCl}$, quirófano y hospitalización en el $94,5 \%$, $88,5 \%, 75,7 \%$ y $76 \%$, respectivamente ( $\leq 0,003)$. La principal indicación fue síndrome anémico $(72,6 \%)$, seguido de hemorragia aguda $(28,6 \%)$, hemoglobina $<7 \mathrm{~g} / \mathrm{dl}$ en paciente crítico $(26,2 \%)$, sepsis $(14,8 \%)$ y hemoglobina $<8 \mathrm{~g} / \mathrm{dl}$ en enfermedad cardiovascular (7,6\%).

Conclusiones. El incumplimiento de indicaciones de transfusiones de glóbulos rojos se presentó en áreas de hospitalización y quirófanos.

\section{Análisis de flujos, tiempos de espera y capacidades en el área de quimioterapia del Centro Javeriano de Oncología}

\author{
Alejandro Ruiz Patiño, Luis Leonardo Rojas Puentes, Laura Elena Acosta Ospina, Annie Natera
}

Centro Javeriano de Oncología, Hospital Universitario San Ignacio, Bogotá, D.C.

Contacto: Irojas@husi.org.co

Introducción y objetivos. Evaluar la capacidad instalada, los tiempos de espera para inicio y el tiempo de ingreso de pacientes en tratamiento en la Unidad de Quimioterapia del Centro Javeriano de Oncología (CJO).

Materiales y métodos. Mediante modelamiento matemático por teoría de colas, se evaluaron las filas formadas en el flujo de pacientes. Con base en la capacidad instalada (23 sillas), se estimó el tiempo de espera para el ingreso una vez el paciente asiste al tratamiento y el tiempo desde valoración por oncología a inicio de quimioterapia (TdQ) para el grupo de pacientes con el diagnóstico de mayor prevalencia entre aquellos en quimioterapia del CJO (cáncer de mama). Se realizaron análisis de sensibilidad con el fin de evaluar el impacto en tiempo de espera en ambas colas que tendría modificar las capacidades.
Resultados. Se obtuvo un flujo máximo de 61 pacientes/día, una tasa de atención de 0,249 pacientes/hora, 23 sillas, una cola de ingreso de 3,7 horas y 34 días para el inicio de tratamiento. Aumentando a 28 sillas, se obtiene una reducción en el tiempo de ingreso al mínimo y la posibilidad de acomodar 7 pacientes/día con cáncer de mama adicionales, lo cual resulta en una reducción del TdQ a 11 días.

Conclusiones. La teoría de colas es una herramienta que permite evaluar las capacidades y tiempos de tratamiento en unidades de quimioterapia, y ayuda a plantear requerimientos locativos y logísticos basados en la capacidad instalada y el flujo de pacientes, buscando mejorar oportunidad y calidad en la atención de pacientes. 